\title{
EL APRENDIZAJE DESDE LA INSTRUCCIÓN: LA EVOLUCIÓN DE LAS ESTRATEGIAS PERSONALES EN TAREAS DE PROPORCIONALIDAD NUMÉRICA
}

\author{
LLINARES CISCAR, S. y SÁNCHEZ GARCÍA, V. \\ Departamento de Didáctica de las Ciencias (Matemáticas). Universidad de Sevilla. Avda. Ciudad Jardín, 22. \\ 41005 Sevilla.
}

\section{SUMMARY}

This study examines the personal strategies evolution used by students in numerical proportionality task solving during instruction. The analysis of a sequence of video/audiotaped lessons allowed the observation of the process of cognitive construction by students in the classroom. The «local» effectiveness of intuitive strategies is considered a phase in the construction of more general strategies.

\section{INTRODUCCIÓN}

En los últimos años, las investigaciones centradas en el estudio de los procesos de enseñanza y aprendizaje de nociones curriculares concretas han mantenido un cierto distanciamiento. Las investigaciones sobre la ensenanza se ocupaban generalmente de los procesos instructivos, sin considerar demasiado la materia específica que ése estaba enseñando. Por otra parte, las investigaciones sobre el aprendizaje se centraban en la estructura del conocimiento de los niños, proporcionando análisis detallados de esa estructura, errores y fases por las que pasaban en su desarrollo sin considerar el proceso de enseñanza.

Estas últimas investigaciones han generado cuestiones relativas a cómo una instrucción determinada influye en la forma en que los niños adquieren estrategias más avanzadas y cómo éstas evolucionan en el seno de las actividades de resolución de problemas. En este contexto, Ios intentos de comprender los cambios cognitivos llevados a cabo durante el proceso instructivo han dado lugar a una serie de estudios sobre cómo los niños aprenden desde la instrucción (Baxter y Stein 1990, Bromme y Steimbring 1990, Carpenter y Peterson 1988, Fennema, Carpenter y Lammon 1988).

El objetivo central de esta agenda de investigación es, precisamente, estudiar las cogniciones de los niños durante la instrucción, para llegar a comprender sus efectos. En esta dirección, se defiende la idea de que el aprendizaje desde la instrucción puede llegar a ser comprendido al considerar la forma en que ésta cambia los procesos cognitivos usados por los estudiantes para resolver determinadas tareas y dotar de significado a las nociones. La construcción mental activa de los estudiantes durante la instrucción es considerada, entonces, el cen* tro de atención (Resnick 1987).

Este problema ha sido abordado desde diversos marcos teóricos que han proporcionado el soporte a las investi. gaciones en esta agenda, originando diseños de investi. gación con rasgos característicos propios.

En particular, desde la perspectiva de las investigaciones sobre la enseñanza del tipo experto/novel, Leinhardt (1987, 1988) describe la adquisición por los niños de diversos tipos de conocimiento matemático (intuitivo, concreto, de cálculo y conceptual) como consecuencia de una secuencia natural de instrucción (la desarrollada por un profesor experto), examinando los cambios en el conocimiento de los estudiantes durante esta secuencia al realizar determinadas tareas instruccionales.

Por otra parte, Hiebert y Wearne (1988), aunque coinciden con Leinhardt en que la comprensión del cambio cognitivo durante la instrucción requiere centrarse en los procesos cognitivos específicos de dominios curricula* res particulares (en particular tópicos concretos del currículo escolar), defienden la idea de que la identifica- 
ción entre procesos instructivos y cambios cognitivos requiere las descripciones detalladas de la instrucción diseñada explícitamente para promover el cambio. En esta dirección, estos autores proponen diversas componentes metodológicas para estudiar el aprendizaje durante la instrucción: 1) Identificar un dominio de contenido bien definido, 2) identificar los procesos cognitivos que son claves para el desarrollo con éxito en este dominio, 3) diseñar una secuencia instruccional que promueva el uso de estos procesos cognitivos, y 4) evaluar los cambios cognitivos a través de las estrategias empleadas en la resolución de determinadas tareas.

Una síntesis de la metodología utilizada en estos estu* dios fue la adoptada en nuestra investigación, en la que se diseñó una secuencia instruccional específica, relativa a tareas de proporcionalidad, que fue desarrollada por un profesor experto (experimentación en el aula en términos de Vergnaud, 1982).

\section{PROPÓSITO DEL ESTUDIO}

El propósito del estudio realizado fue:

Analizar los procesos de construcción activa de estrategias de resolución en tareas de proporcionalidad numérica, desarrollados por estudiantes de séptimo de EGB, en una secuencia instruccional especifica.

Este propósito inicial se concretó en los siguientes puntos:

a) Identificar las estrategias empleadas por estudiantes de séptimo de EGB para resolver tareas de proporcionalidad numérica.

b) Describir cómo dichas estrategias evolucionan durante la generación de diferentes procesos de resolución.

c) Analizar la naturaleza de los cambios realizados en las estrategias empleadas.

d) Determinar las características de la secuencia instruccional que estén vinculadas a los cambios en dichas estrategias.

\section{DESCRIPCIÓN DEL ESTUDIO REALIZADO}

\section{Sujetos}

El estudio se llevó a cabo con 36 niños de séptimo curso de EGB (rango de edades: 12-13 años) que no habían recibido, previamente a la experiencia, ningún tipo de instrucción relacionada con los "problemas de regla de tres».

\section{Diseño}

Se siguieron las indicaciones de Hiebert y Weame (1988). En ese sentido: a) Se identificó como dominio de contenido algunas tareas de proporcionalidad numérica. El contexto lo proporcionaron las relaciones entre la longitud de una va. rilla y la longitud de la sombra producida al colocarla al sol en posición vertical al suelo. En las tareas numéricas, los datos iniciales pertenecían al dominio de los números naturales. Las tareas escritas pertenecían al tipo de «situaciones con incógnita» (problemas de regla de tres) $\mathrm{y}$ «situaciones de comparación». Los operadores funcionales considerados $[f(x)=a x$ ] fueron $3,3 / 2$, y $4 / 3$ (fig. 1 ).

\section{Figura ]}

Tablas de doble entrada propuestas en el contexto de las relaciones entre las longitudes de las varillas y las longitudes de sus sombras.

a) Completar y explicar cómo se ha hecho.

\begin{tabular}{c|c} 
long. V & long. S \\
\hline 2 & 6 \\
6 & 12 \\
8 & \\
& 36
\end{tabular}

b) Buscar la relación numérica entre las dos columnas que permita obtener un dato en la segunda, conociendo el de la primera (y viceversa).

\begin{tabular}{c|c} 
long. V & long. S \\
\hline 2 & 3 \\
4 & 6 \\
6 & 9 \\
10 & 15
\end{tabular}

c) Lo mismo que en b).

\begin{tabular}{c|r} 
long. $V$ & long. S \\
\hline 3 & 4 \\
6 & 8 \\
9 & 12 \\
12 & 16
\end{tabular}

b) Se identificaron los procesos cognitivos caracteristicos en este dominio ${ }^{\dagger}$. Las investigaciones previas sobre el aprendizaje del razonamiento proporcional nos han aportado descripciones de diferentes estrategias empleadas por los niños y, por tanto, de las diferentes características de la comprensión de las relaciones de proporcinalidad (Dupuis y Pluvinage 1981, Hart 1981, Karplus et al. 1983, Koch 1987, Lawson y Wollman 1980, Tournaire 1986, Tournaire y Pulos 1985, Vergnaud 1983, Romberg, Lamon y Zarinnia 1988),

c) Diseño de una secuencia instruccional ${ }^{2}$. El diseño instruccional fue desarrollado durante ocho sesiones en un período de un mes de clase. Estaba articulado a través del trabajo en pequeños grupos (de cuatro o cinco estudiantes), en los que se favorecía el diálogo colaborativo 
y la resolución de puntos de vista conflictivos. Los trabajos en pequeños grupos se alternaban con discusiones con la clase entera en las que cada grupo explicaba los procedimientos aplicados para resolver las tareas.

En este contexto, cuando los estudiantes intentan «comprender» los procesos de resolución del problema desarrollados por otro, amplían su marco conceptual al construir un dominto consensuado con su compañero. Es decir, esta característica del diseño instruccional se apoyaba en la idea de que el lenguaje puede ayudar a reflexionar al estudiante sobre su propia comprensión cuando da explicaciones, ayudándole a reconceptualizar sus propias construcciones cognitivas (Onslow 1988, Perret-Clermont 1984, Wood y Yackel 1990).

Las características generales del diseño instruccional (fig. 2) pretendían proporcionar a los estudiantes más autonomía en su aprendizaje, generando oportunidades de mirar las relaciones numéricas desde sus propios puntos de vista, así como posibilitar una forma personal de buscar una respuesta a las cuestiones planteadas.

d) La valoración de los cambios cognitivos. La dinámica de la secuencia instruccional llevaba integrados aspectos de evaluación (representados por las cuestiones planteadas por el profesor) atendiendo a la naturaleza de las relaciones numéricas establecidas entre los datos, lo que nos permitía determinar la forma en que se modificaban o no los procedimientos empleados para adecuarse a la estructura de la nueva situación.

\section{Instrumentos de recogida de datos}

Se utilizó una cámara de vídeo para grabar las interacciones en un grupo de trabajo y cuando se realizaban las discusiones con la clase entera. Por otra parte, las interacciones en los otros grupos reducidos fueron grabadas en audio.

Las cintas de vídeo y audio obtenidas se transcribieron y analizaron posteriormente, lo que nos permitió obtener descripciones de cómo los estudiantes resolvían cada tarea en el seno de los grupos, y en la clase entera; y cómo las interacciones entre ellos y el profesor, en su papel de tutor, originaban los cambios de estrategias empleadas.

\section{RESULTADOS}

Los resultados que presentamos son una selección del análisis de una serie de protocolos que muestran la evolución de las estrategias ${ }^{3}$ empleadas por cuatro niños de un grupo de trabajo. Se presenta el análisis de las interacciones desarrolladas en pequeño y gran grupo al tratar con una de las últimas tareas de la secuencia de instrucción (fíg. 1)

Los protocolos descritos en primer lugar (1 y 2) conesponden al trabajo en grupos reducidos en relación a las tareas $b$ ) y c) de la figura 1, y el protocolo 3 corresponde al momento en que los niños de este grupo comunican a sus compañeros la estrategia utilizada para resolver la tarea a) con la discusión generada. Los protocolos se presentan cronológicamente según se desarrollaron.

\section{A) Protocolo 1}

En el grupo de trabajo que estamos considerando, después de una discusión previa entre sus miembros en relación a la tarea b) de la figura 1, Estrella, uno de los miembros, describe la siguiente estrategia aceptada como válida por todos.

$$
\begin{array}{r|cl}
2 & 3 & \text { Estrella: }[. . .] \text { a la primera columna, al } \mathrm{n}^{9} \text { de la } \\
4 & 6 & \text { primera columna se le sumaría } 1 ; 2 \text { y } 1 \text {, tres } \\
6 & 9 & \text { al siguiente se le sumaría } 2 ; 2 \text { y } 4 \text {, seis; } \\
10 & 15 & \text { al siguiente se le sumaría } 3 ; 6 \text { y } 3 \text {, nueve; } \\
& \\
& \text { al siguiente se le sumaría } 4, \text { pero falta aquí; } \\
\text { y al otro } 5 ; 10 \text { y } 5 \text {, quince... }
\end{array}
$$

En este primer momento, la estrategia común defendida por el grupo constituye lo que Hart (1981) denomina un modelo numérico:

\begin{tabular}{rl|l|l}
2 & + & 1 & $=$ \\
4 & + & 2 & $=$ \\
6 & + & 3 & $=$ \\
8 & + & 4 & $=$ \\
10 & + & 5 & $=$
\end{tabular}

Las dos características de esta estrategia estarian en que:

i) colocan los números que faltan, en este caso el 8 en la primera columna, para mantener la constante $(+2)$ en la primera columna, y

ii) asocian «el siguiente a» en la primera columna a la constante $(+1)$. Existe un modelo implícitoen relación al orden, en este caso con una constante $(+2)$ en la primera columna.

En estos momentos el profesor introduce nuevos datos en la primera columna, con el propósito de buscar la generalización de la estrategia y el posible conflicto: 
Figura 2

Fases de la secuencia de enseñanza.

\begin{tabular}{|c|c|}
\hline $\begin{array}{l}\text { PRESENTACIÓN } \\
\text { * Actividad de clase entera, } \\
\text {. Presentación de la tarea. } \\
\text {. Debate. Determinar posibles variables que influyen a la hora de } \\
\text { calcular la longitud de la sombra de una varilla (hora, posición } \\
\text { varilla, longitud varilla...). }\end{array}$ & * Cuestiones formuladas por el profesor y los propios aiumnos. \\
\hline $\begin{array}{l}\text { EXPERIMENTACION } \\
\text { * Trabajo en grupo ( } 4-5 \text { niños). } \\
\text {. Recogida de datos de forma empírica al colocar varillas de } \\
\text { diferente longitud al sol. }\end{array}$ & * Trabajo de campo (recogida de datos en el patio). \\
\hline $\begin{array}{l}\text { REPRESENTACIÓN } \\
\text { " Trabajo en grupo. } \\
\text { Organizar y tabular los datos. } \\
\text {. Construir gráficas. }\end{array}$ & * Interacciơn alumno-aiumno. \\
\hline $\begin{array}{l}\text { COMUNICACIÓN } \\
\text { * Actividad de clase entera. } \\
\text { Se ponen en común los diferentes datos obtenidos por los grupos. } \\
\text { Se elige una tabla. Se buscan relaciones entre los datos. } \\
\text {. Intentos de generalización de Jas relaciones obtenidas introdu- } \\
\text { ciendo nuevos datos. }\end{array}$ & $\begin{array}{l}\text { * El profesor plantea cuestiones que faciliten la evajuación y } \\
\text { comparación de las relaciones encontradas. } \\
\text { * Se identifican cuestiones sin solución } \\
\text { * Se identifican algunas estrategias que resuelven algunos casos } \\
\text { pero de dificil aplicacion en otros. }\end{array}$ \\
\hline $\begin{array}{l}\text { REFLEXIÓN } \\
\text { * Actividad de clase entera. } \\
\text { Idertificar otros contextos en los que también aparecen las } \\
\text { mismas relaciones. } \\
\text { (Ej.: Artículo/precio...) }\end{array}$ & * Traslación. \\
\hline $\begin{array}{l}\text { EXPERIMENTACIÓN } \\
\text { * Trabajo en grupo. } \\
\text {. Realización de hojas de actividades con tareas estructuralmente } \\
\text { parecidas a las desarrolladas hasta el momento (fig. 1). }\end{array}$ & $\begin{array}{l}\text { * Interacción alumno-alumno. } \\
\text { * Tutoría del profesor: cuestiones dirigidas a evaluar la efectividad } \\
\text { de la estrategia empleada. }\end{array}$ \\
\hline $\begin{array}{l}\text { COMUNICACION } \\
\text { * Actividad de clase entera. } \\
\text { Se verbalizan las estrategias empleadas en cada grupo para } \\
\text { resolver las tareas. }\end{array}$ & * Profesor: esquematizar/resumir propuestas. \\
\hline $\begin{array}{l}\text { REFLEXION } \\
\text { * Actividad de clase entera. } \\
\text { Debate sobre la efectividad de las estrategias empleadas. } \\
\text {. Correspondencia: tinitaciones/condiciones estrategia y caracte- } \\
\text { rísticas estructurales. } \\
\text { Síntesis de las estrategias. }\end{array}$ & $\begin{array}{l}\text { * Interacción alumno-alumno: identificat conflictos, evaluar la } \\
\text { efectividad. }\end{array}$ \\
\hline
\end{tabular}

La dificultad de análisis planteada en este protocolo se deriva del hecho de que no se dan explicaciones de las respuestas proporcionadas al no pedírselas el profesor. La estrategia parece adaptarse a los nuevos datos. Dos explicaciones alternativas se pueden generar ${ }^{4}$ :

i) Que se estén utilizando «isomorfismos aditivos»

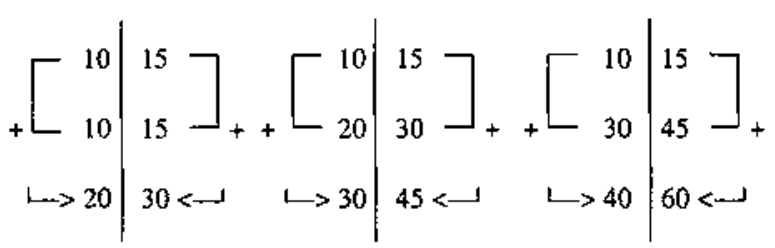


ii) Otra posible explicación es que sí al 10 en la primera columna se le sumaban 5 para pasar a la segunda,

$$
10+5=15
$$

si multiplicamos por dos el número de la primera columna (es decir, si lo doblamos) también se debería muitiplicar por dos la constante que se suma.

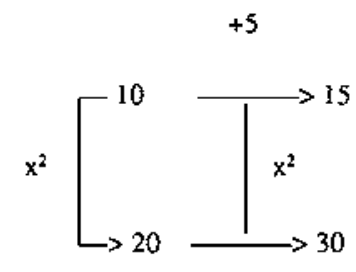

Así, parece modificar la estrategia empleada hasta ahora para adecuarla a la nueva situación.

Se siguen introduciendo nuevos datos por el profesor, originándose un diálogo entre los miembros del grupo.

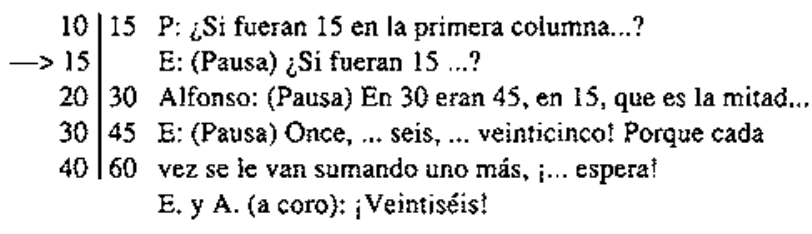

Inicialmente existe una interacción entre dos niños del grupo (Estrella y Alfonso). Alfonso intenta utilizar «cálculo de mitades", aprovechando datos conocidos. Constituye una primera aproximación al operador escalar multiplicativo (aunque en una primera aproximación sólo es para mitades y dobles). Esta aproximación coincide con los resultados de investigaciones previas en el sentido de que las razones $2: 1(1: 2)$ son utilizadas en una primera fase del desarrollo del razonamiento proporcional.

Sin embargo aparece una dificultad adicional al tener que calcular la mitad de un número impar. Esta dificultad de cálcuio induce a Alfonso a abandonar su estrategia para «apoyar» la anterior del «modelo numérico» que parece más seguro, rellenando a partir del número diez en la primera columna los datos que faltan hasta llegar al 15 .

\begin{tabular}{r|rl}
2 & 3 & E: Son veinticinco. \\
4 & 6 & P: ¿Por qué? \\
6 & 9 & E: (Pausa) Porque... son cinco más... \\
10 & 15 & de 12,7 más; \\
$\rightarrow 15$ & & de 13,8 más; \\
20 & 30 & de 14,9 más; \\
30 & 45 & de 15,10 más; \\
40 & 60 & y 10 y 15, veinticinco!
\end{tabular}

En la aplicación en esta nueva situación de la estrategia que hemos denominado "modelo numérico», aceptada como estrategia idónea por el grupo, se comete un error.

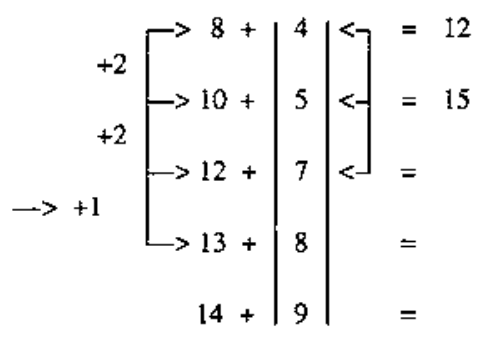

Ahora los números que se tiene que rellenar son demasiados, lo que ha hecho que el "modelo implícito» del orden de los números naturales haya podido influir en la modificación de la constante $(+2)$ cambiándola por $(+1)$ en la primera columna. Unido a esto se encuentra el hecho de que el número 15 es impar, lo que puede haber inducido a empezar a considerar números impares también en la primera columna para poder «alcanzario».

Esta «modificación» de la estrategia primitiva se afianza en las nuevas situaciones introducidas por el profesor.

$$
\begin{array}{r|rl}
2 & 3 & \text { P: ¿Y 12 en la primera columna? } \\
4 & 6 & \text { A: (Pausa) ¿18? } \\
6 & 9 & \text { P: ¿Por qué? } \\
10 & 15 & \text { E: i... 19! } \\
& \text { P: ¿Por qué? } \\
& \text { E: Porque de } 10 \text { son } 5 \text { más; } \\
& \text { de 11, seis más; } \\
& \text { de } 12, \text { siete más. }
\end{array}
$$

A partir de aquí, la interacción entre los miembros del grupo hace que aparezca otra estrategia, identificada por Ricco (1982) como las «diferencias constantes». Esta nueva estrategia es aceptada como correcta por su coherencia con la situación, haciendo que Estrella modifique la suya y, por tanto, su respuesta.

\begin{tabular}{r|r}
2 & 3 \\
4 & 6 \\
6 & 9 \\
10 & 15
\end{tabular}

Pablo: (señalando la segunda columna). Van de

3 en 3.3 y 3,$6 ; 6$ y 3,$9 ; \ldots$ pero, ¿aquí?

Alfonso: iría el 12!

Pablo: iEs verdad! Aquí se han saltado un número, el 8. Aquí vendría el 8 (señalando en la primera columna... el doce... ;hacían 18, sít

Profesor: ¿Seguro que es 18 ?

Alfonso: ¡Śíl Sí porque... van de tres en tres (señalando en la segunda columna). Detrás del 9 viene el 12 ; y 15 más 3 son 18 .

Estrella: ¡Es verdad!

En estos momentos la interacción entre los niños ha hecho que se abandonara una estrategia (modelo numé- 
rico) y se aceptara otra (diferencias constantes) para dar respuesta a la situación planteada. Una característica de esta nueva estrategia utilizada es que la atención de los niños recae en la segunda columna y en la constante que conlleva, $(+3)$, y no se menciona la primera columna.

Este hecho coincide con la idea de que en una primera fase del desarrollo del razonamiento proporcional la atención de los niños sólo recae sobre una de las variables y no se coordinan las dos variables que están manejando (longitud varilla / longitud sombra). En este caso sería la coordinación entre la variación $(+2)$ en la variable longitud de varilla y la variación $(+3)$ en la longitud de la sombra.

La no coordinación entre las variaciones en las dos variables $[(+2)$ en la primera columna y $(+3)$ en la segunda] se pone de manifiesto inmediatamente después.

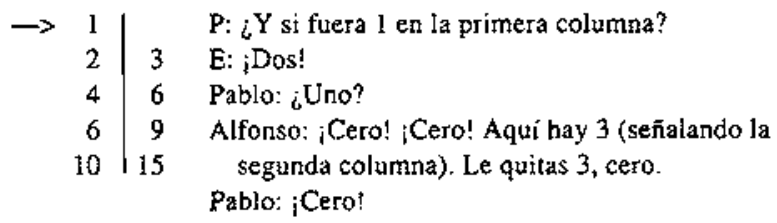

Dos características hay que resaltar en esa situacion.

i) La aplicación sistemática de la constante $(+3)$ en la segunda columna no relacionada con la constante $(+2)$ en la primera columna. Este hecho también ha sido puesto de manifiesto por Ricco.

$$
\left.\begin{array}{lll|l}
\rightarrow & +1 & {\left[\begin{array} { l | l } 
{ - 1 } & { < } \\
{ } & { + 2 }
\end{array} \quad \left[\begin{array}{l}
3<-3 \\
-4
\end{array}\right.\right.} & 6<-
\end{array}\right]+3
$$

ii) Por otra parte, en esta situación se ha añadido una dificultad adicional al tener que considerar como posible respuesta una fracción.

En este caso la "potencia» del modelo sumar (restar) la constante (+3), muy semejante al modelo implícito del orden de los números naturales (sumar la constante $(+1)$ ) ha evitado cualquier replanteamiento de la estrategia que hubiese podido llevar a considerar la posibilidad de la introducción de una fracción como respuesła. Así, se introduce una respuesta que puede tener poco sentido en el contexto de las longitudes en que se trabajaba.

Este análisis nos conduce a cuestionarnos si la introducción del procedimiento «reducción a la unidad» en la enseñanza de los problemas de proporcinalidad directa, que se suele emplear en las escuelas, no debería estar vinculado a:
- la estrategia de resolución que estén empleando los niños en esos momentos, y

- la aparición o no de fracciones/decimales como respuesta.

En estos momentos desconocemos si existe algún estudio que indique la existencia (o no) de una correlación significativa entre estos dos factores, y la frecuencia de éxito en la utilización por los niños de esta aproximación en la resolución de problemas de proporcionalidad directa (regla de tres).

\section{B) Protocolo 2}

El análisis presentado en esta sección corresponde a las interacciones registradas en el trabajo realizado con la tabla de la figura 1 (c) que se desarrolló a continuación de la tarea anterior. La primera estrategia (modelo numérico) es reconocida como válida por Alfonso.

$$
\begin{array}{r|rc}
3 & 4 & \text { Alfonso: iÉsa es igual! (refiriéndose a la } \\
6 & 8 & \text { otra tabia) } 3 \text { y } 1 \text {, cuatro; } 6 \text { y } 2,0 \text { ocho: } \\
9 & 12 & 9 \text { y } 3, \text { doce. } \\
12 & 16 &
\end{array}
$$

Se reproduce en estos momentos la estrategia «modelo numérico» empleada en la tabla anterior

$$
\begin{aligned}
& 3+\square 1 \\
& 6+2 \\
& 9+\sqrt{2}=4
\end{aligned}
$$

Existe una fuerte insistencia en asociar «el siguiente número» en la primera columna a «uno más». El modelo implícito del orden đe los números naturales puede estar influyendo en estos momentos. Este hecho general de la aplicación «del siguiente a» también ha sido puesto de manifiesto por Wollman y Lawson (1978) aunque en otro contexto algo distinto. Estos autores lo explican diciendo que probablemente el uso de esquemas «for every» proceda del esquema $I: I$, anteriormente desarrollado y ya conceptualizado que subyace en el contar. Estaría en este caso haciendo la función de un obstáculo epistemológico.

Por otra parte, la generalidad de la estrategia de las «diferencias constantes» (en relación con la estructura de los problemas planteados) viene dada por el hecho de que la diferencia entre las variaciones en la primera columna [en este caso $(+3)$ y en el anterior $(+2)$ ] no influye para nada en su aplicación.

Ante la nueva cuestión planteada, que altera la estructura de la situación manejada hasta este instante, se realiza un avance «cualitativo» en la evolución de las estrategias. Se empiezan a coordinar las variaciones en las dos variables. 


\begin{tabular}{r|rl}
3 & 4 & P: ¿Cuál sería la imagen del 15? \\
6 & 8 & Estrella: Aquí van de 4 en 4 (señalando la \\
9 & 12 & columna) (Pausa) ¡El 20! \\
12 & 16 & P: ¿Del 15 al 20? \\
& & Alfonso: A ver un momento... ¡Sí claro! \\
$\rightarrow 15$ & & Porque 12 y 3 son 15, y 16 más 4, veinte.
\end{tabular}

Aquí Alfonso coordina la variación $(+3)$ en la primera columna con $(+4)$ en la segunda

$$
+3\left[\begin{array}{cc|cc}
9 & 12 & \\
> & 12 & 16 & < \\
> & 15 & <
\end{array}\right]+4
$$

Una vez conseguida la coordinación entre las variaciones de las dos columnas de la tabla, la evolución de la estrategia utilizada hacia el «isomorfismo aditivo» $[f(a)+f(b)=f(a+b)]$ es muy rápida.

\footnotetext{
$12 \mid 16$ P: ¿Si fueran 60 en la primera columna?

1520 Alfonso: (Pausa) Sumando 15 y 15 son treinta, y como 15 son veinte (refiriéndose a su imagen), como 30 es el doble (señalando la segunda columna), también tiene que ser el doble... de 60 ... ochenta.

P: ¿Puedes explicar cómo lo has hecho?

A: Porque el doble de 15 es 30 , y entonces si es 30 , tiene que ser el doble también aquí (señalando la segunda columna) de 30 son $40 \ldots$ de $45 \ldots$ veinte más (señalando la segunda columna) son 60 ... y de $60 \ldots$ ochenta.
}

En un primer momento Alfonso utiliza el operador «doble» para construir la respuesta por «segmentos». La utilización de la relación «doble de» para ir acercándose a la respuesta de la cuestión es una aproximación primitiva de la estrategia que Hart ha denominado «construcción de la respuesta». Inicialmente se emplea la estrategia «uso de mitades» (en nuestro caso dobles) cuando los niños emplean la información que ellos han obtenido en f́tems anteriores para generar la respuesta pedida.

Sin embargo, al pedir una explicación del proceso seguido, Io readapta para justificar la respuesta a través de la estrategia "isomorfismo aditivo», lo que indica la «proximidad» cognitiva entre estos dos procedimientos. Este hecho indica realmente que la estrategia de ir acercándose a la respuesta mediante relaciones aditivas en cada columna, como ha sido utilizada en esta situación, puede ser vista como un caso particular de la estrategia más elaborada denominada «isomorfismo aditivo", en el sentido de exigír esta última menos condiciones para ser efectiva.
De la coordinación de las variaciones en las dos columnas se ha derivado hacia la utilización del isomorfismo aditivo, con un paso intermedio en la utilización de dobles.

$+15\left[\begin{array}{llll}15 \\ +15\end{array} \begin{array}{llll}15 & 20 & < \\ 30 & 40 & < \\ 45 & 60 & < \\ 60 & & <2\end{array}\right] \begin{aligned} & \times 20 \\ & +20\end{aligned}$

\section{C) Protocolo}

Este protocolo corresponde a la fase final de la sesión de trabajo, cuando se comunica a la clase entera el proceso de resolución seguido con cada una de las tareas desarrolladas y las respuestas producidas. Este tipo de actividad de comunicar, tanto las estrategias como los resultados al resto de los compañeros, pone de manifiesto la existencia de diferentes niveles de elaboración en las estrategias empleadas en los grupos de trabajo. Esto da pie a la posibilidad de que se produzcan «conflictos» planteados por los propios compañeros ante la idoneidad o no de determinadas estrategias, para responder a ciertas cuestiones. Se plantean en estos casos preguntas relativas a la generalidad de las estrategias empleadas, en el sentido de su efectividad ante diferentes condiciones estructurales de la tarea.

El protocolo siguiente intenta mostrar una de estas situaciones. El profesor en esta actividad se encuentra en la pizarra, organizando la discusión y resumiendo las diferentes estrategias verbalizadas correspondientes a la tarea generada por la tabla a) de la figural.
Aquí los niños están reproduciendo la estrategia de las "diferencias constantes», coordinando las variaciones en las dos columnas. En esta situación en particular esta estrategia se revela eficaz. 


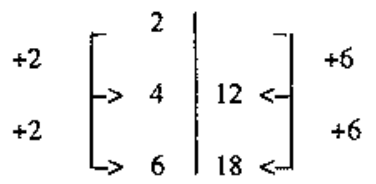

La eficacia de esta estrategia está vinculada a las características particulares de esta situación, como puede ser el que se puede «llegar» a los datos que se piden por ser números pares y relativamente pequeños. Su ineficacia se pone de manifiesto cuando la longitud de la varilla que se proporciona no cumple estos requisitos. Es decir, la generalidad de la estrategia está limitada por estas condiciones como pone de manifiesto Rafael, un niño que había estado trabajando en otro grupo donde habían empezado a utilizar operadores funcionales (relación entre el origen y la imagen; $a=f(x) / x$ ). En este caso, el operador funcional es un número natural (x3), lo que favorece precisamente el empleo de operadores funcionales a diferencia de las otras tareas (tareas b y c, figura 1) en donde dicha relación era una fracción.

La no generalidad de la estrategia de las diferencias constantes se pone de manifiesto por la intervención de Rafael.

\begin{tabular}{|c|c|c|}
\hline 2 & 6 & \\
\hline 4 & 12 & \\
\hline 6 & 18 & \\
\hline 8 & 24 & $\begin{array}{l}\text { Rafael: ¿Y si fuera, por ejemplo, el } 25 \text { en } \\
\text { la primera columna? }\end{array}$ \\
\hline 10 & 30 & $\begin{array}{l}\text { Alfonso: (Pausa) A } 14 \ldots 42, \ldots \\
\text { (Pąusa prolongada) }\end{array}$ \\
\hline 12 & 36 & \\
\hline & & \\
\hline
\end{tabular}

Alfonso intenta seguir aplicando su estrategia, coordinando las variaciones $(+2)$ y $(+6)$ en las dos columnas, y "rellenando" los datos que faltan. Evidentemente esta estrategia ya se ha manifestado no del todo idónea para resolver esta cuestión. Junto al hecho de que el 25 es un número que no se «alcanza» con la serie de números pares, que constituyen las primeras columnas de esta tarea, está el hecho de tener que «rellenar» muchos números. Sin embargo, se pone de manifiesto la insistencia por parte de los niños en la utilización de determinadas estrategias, que han sido correctas en determinadas situaciones, aunque ya no sean válidas, ante la modificación de alguna de las características estructurales de la tarea inicial.

Ante la insistencia de Alfonso en aplicar «su» estrategia, Rafael apunta:

Rafael: ¡Y si fuera el 1.125!
Aquí se plantea un conflicto. El tamaño del número hace que se cuestione la idoneidad de la estrategia empleada hasta este momento. La situación planteada y las nuevas propuestas que se originan señalan la posibilidad de la utilización de los operadores escalares

$$
\mathrm{xk} \quad\left[\begin{array}{l|l}
\mathrm{a} & b \\
\mathrm{c} & <
\end{array}\right] \mathrm{xk}
$$

y su introducción como una «estrategia resumida» de la estrategia de las diferencias constantes. Estrella, la niña del grupo del que hemos estado analizando las interacciones, pide la palabra y señala:

$$
\begin{array}{r|rc}
2 & 6 & \\
4 & 12 & \\
6 & 18 & \text { Estrella: [...]Dividiendo } 1.125 \text { entre } 2 \text { y lo } \\
8 & 24 & \text { que dé se multipitica por } 6 \ldots \text { y da... } \\
12 & 36 & \\
& &
\end{array}
$$

Esta estrategia es lo suficientemente general al permitir resolver cuestiones que resultaban difíciles de resolver por las otras.

\section{DISCUSIÓN}

La evolución en las estrategias empleadas en la resolución de las tareas planteadas, desde unas con una eficacia «local» hasta otras de eficacia más general, muestran un camino hacia la comprensión (conocimiento conceptual) de las relaciones de proporcionalidad numérica (Tabla I).

El análisis presentado en este trabajo pretende mostrar los aspectos dinámicos del aprendizaje en tareas de proporcionalidad numérica. Dos aspectos caben resaltar.

En primer lugar las características del diseño de la secuencia de enseñanza, que favorecían el que los estudiantes se enfrentaran a las tareas instruccionales desde sus propios puntos de vista, y el papel determinante desempeñado por el hecho de tener que verbalizar las estrategias empleadas en su resolución, permitiendo su reconceptualización. En esta situación, los momentos de proporcionar explicaciones o de escuchar al compañero, se constituyen en oportunidades para aprender, al participar en la elaboración de la estrategia más efectiva, como se ha mostrado en los protocolos anteriores.

Por otra parte, desde el punto de vista de las estrategias empleadas por los estudiantes en la resolución de las tareas de proporcionalidad planteadas, hay que resaltar la transición experimentada por éstas desde la eficacia «local» a una eficacia más general, que se correspondía 
Tabla I

Estrategias. Características y jerarquía.

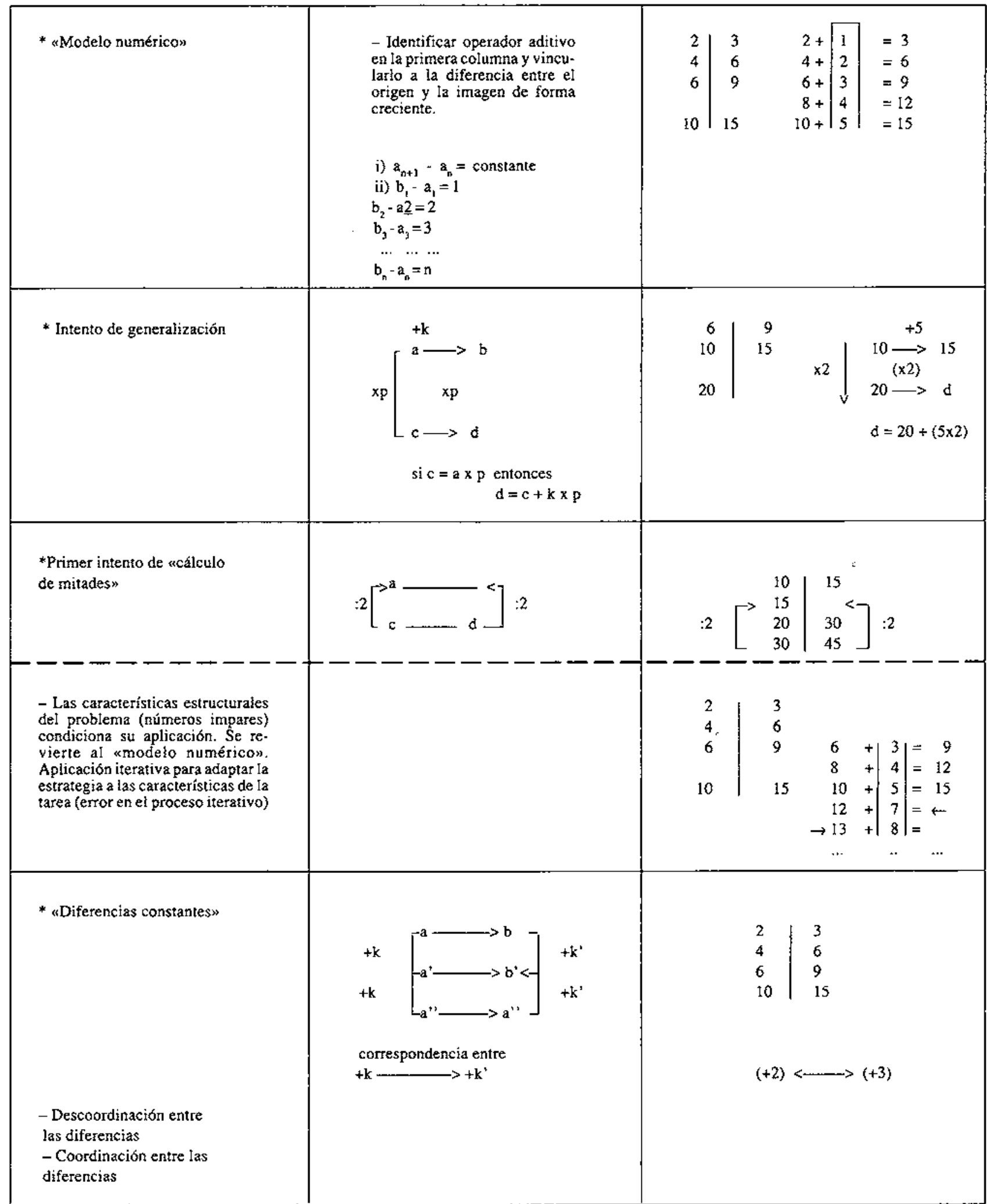


Tabla I (continuación)

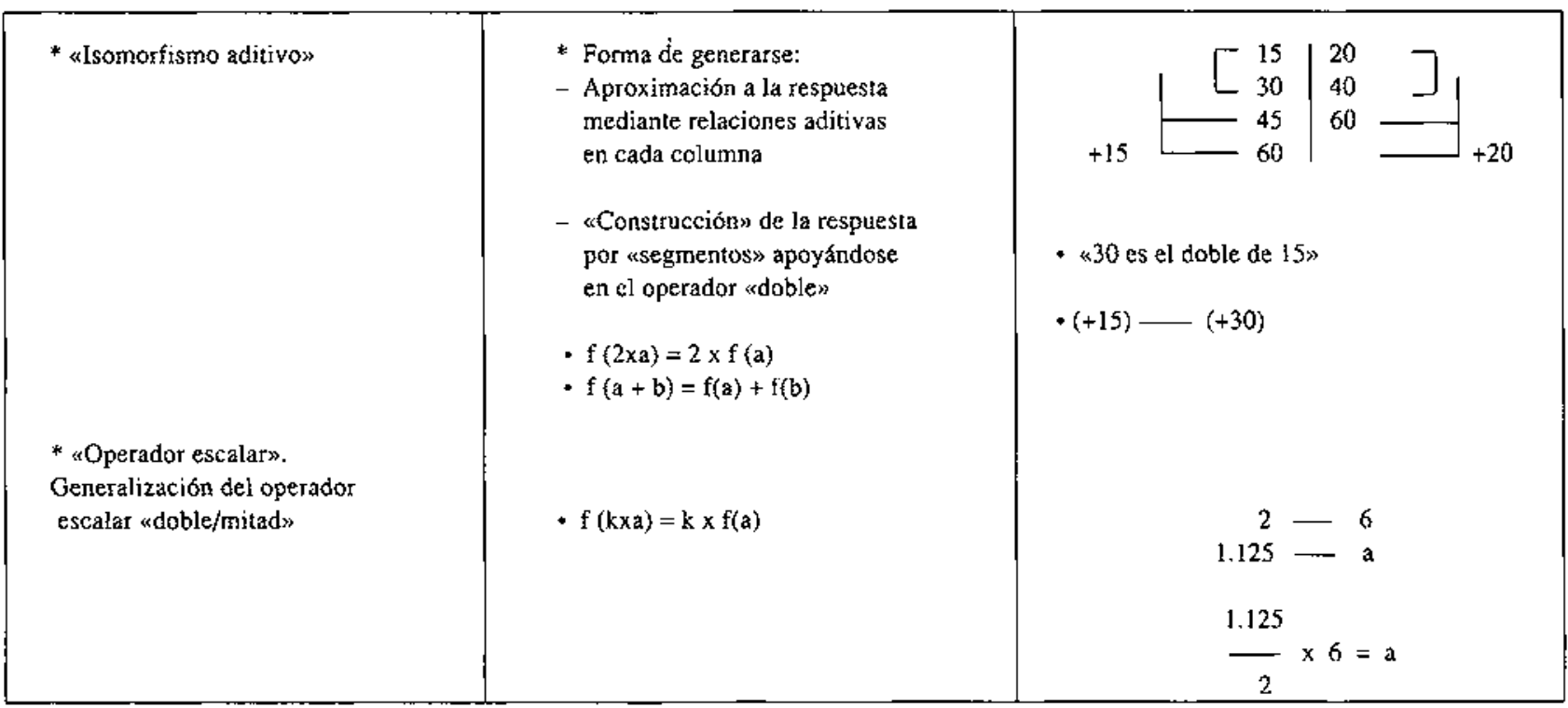

a una comprensión más global de todas las variables y relaciones numéricas implicadas.

Desde el empleo de la estrategia denominada «modelo numérico», pasando por el proceso de «ir construyendo la respuestan (usando dobles y mitades) al coordinar las diferencias constantes, hasta el empleo del «isomorfismo aditivo" y los operadores escalares y funcionales, que nos aproximaban a las bases del futuro algoritmo de la regla de tres, se opera un cambio en los procesos cognitivos de los estudiantes, en el sentido de ir adaptando las estrategias empleadas en un primer momento a las características estructurales de las nuevas situaciones, produciéndose los «saltos cualitativos» que caracterizan este aprendizaje.

En este sentido, la evolución de las estrategias emplea* das viene definida por la existencia o no de una correspondencia entre las características estructurales de la tarea y las condiciones/limitaciones inherentes a una estrategia concreta. Desde esta perspectiva, hay que centrarse en esta correspondencia para poder comprender cómo dichas estrategias evolucionan durante la ge* neración de los diferentes procesos de resolución.

Así, podemos considerar que las intervenciones del profesor sólo pueden ser consideradas como generado* ras de la evolución de las estrategias si modifican, de alguna manera, la correspondencia entre las condiciones de funcionamiento de la estrategia y las características estructurales de la tarea (conocimiento de contenido pedagógico del profesor).

La efectividad «local» de las primeras estrategias empleadas no debe considerarse como consecuencia de ser erróneas, sino más bien como etapas necesarias en la construcción posterior de las aproximaciones sucesivas a una estrategia más general, que es considerada, desde esta perspectiva, como síntesis de las estrategias personales evolucionadas.

Hay que indicar que los cambios en las estrategias utilizadas por los estudiantes presentadas aquí no deben sacarse de su contexto ni separarse del tipo de actividades concretas utilizadas. Sin embargo, habria que considerar, en futuros estudios sobre el aprendizaje desde la instrucción de nociones relativas a la proporcionalidad, el paralelismo encontrado en la secuencia presentada con las estrategias identificadas en otras investigaciones empíricas (Hart 1981, Ricco 1982) basadas en la elaboración de cuestionarios.

Este trabajo inicial también presenta cuestiones de índole metodológico. La posible introducción de entrevistas clínicas utilizando recursos como la «estimación del recuerdo» durante el desarrollo de la secuencia instruccional y a su término deberán aportar información que permita obtener respuesta a cuestiones derivadas del análisis de los protocolos presentados.

El problema, desde esta nueva perspectiva de la vinculación entre la instrucción y el aprendizaje sigue abierto. necesitándose más estudios que impliquen diferentes contextos, magnitudes, clase de razones empleadas, modificación de la presentación de las tareas, etc.

Finalmente cabría señalar que este tipo de estudios pretenden aportar información en relación a la manera de analizar el aprendizaje, desde un punto de vista dinámico, producido como consecuencia de una instrucción determinada. 


\section{NOTAS}

1. Los procesos cognitivos del estudiante se infieren de las respuestas a las tareas (estrategias empleadas) ya que se suponen que son "procesos internos" e indican un razonamiento apoyado en la posible comprensión de las relaciones de proporcionalidad numérica.

2. Para Hiebert y Wearne se deberia esperar una mejora en la realización de las tareas detectadas al observar Ias estrategias de solución empleadas desde las que se puede inferir el uso de los procesos cognitivos identificados como "claves" para resolver con éxito las tareas de proporcionalidad numérica.

$$
\begin{gathered}
f(x+y)=f(x)+f(y) \\
f(x)=a . x
\end{gathered}
$$

\begin{tabular}{c|c}
$\mathrm{d}$ & $\mathrm{f}(\mathrm{x})$ \\
$\mathrm{n}$ & $\mathrm{f}(\mathrm{y})$ \\
$\mathrm{x}+\mathrm{y}$ & $\mathrm{f}(\mathrm{x})+\mathrm{f}(\mathrm{y})<$
\end{tabular}

\section{REFERENCIAS BIBLIOGRÁFICAS}

BAXTER, J.A. Y STEIN, M.K., 1990. Linking Teaching and Learning Through Representations: A case from Functions and Graphing. Paper of Leaming Research and Development Center. (University of Pittsburgh).

BROMME,R. y STEINBRING,H. 1990. Interactive Development of Subject Matter within Instruction in the Classroom. Paper of Institu\& für Didaktik der Mathematik. (Universität Bielefeld).

CARPENTER, T. y PETERSON, P., 1988. Learning through Instnuction: The Study of Students' Thinking during Instruction in Mathematics, Educational Psychologist, 23(2), pp. $75-89$.

DUPUIS, C. y PLUVINAGE, F., 1981. La proportionalité et son uțilisation, Recherches en Didactique des Mathématiques, 2(2), pp. 165-212.

FENNEMA, E., CARPENTER, T.P. y LAMON, S., 1988. Integrating Research on Teaching and Learning Mathematics. (NCRMSE: Madison).

HART, K., 1981. Ratio and Proportion, en K. Hart (ed.), Childrens' Understanding of Mathematics, 11-16. (Murray: London).
3. En este trabajo se denomina estrategia al procedimiento o método empleado por los niños para resolver con éxito las tareas de proporcionalidad numérica.

4. Otra posible conjetura sería que tras la construcción del par $20-->30$ mediante la estrategia $x^{2}$, los niños reconozcan la secuencia $10,20,30,40$ como una variante de la secuencia $2,4,6,8,10$ y que utilicen la estrategia inicial del "modelo numérico" adaptada a los nuevos incrementos $(+10 \mathrm{y}+15)$.

\section{RECONOCIMIENTOS}

Agradecemos el apoyo prestado en las distintas fases de la realización de este estudio a nuestros compañeros Carmen Pereda, Margarita Garrudo y José Antonio Rivero.

HIEBERT, J. y WEARNE, D., 1988. Instruction and Cognitive Change in Mathematics, Educational Psychologist, 23(2), pp. 105-117.

KARPLUS, R., PULOS, S. y STAGE, E.K., 1983. ProportionaI Reasoning of Early Adolescent, en R. Lesh y M. Landau (eds.), Acquisition of Mathematics Concepts and Processes. (Academic Press: London).

KOCH, L., 1987. Strategies Used by College Students Enrolled in Development Mathematics to Solve Proportional Reasoning Problems. Paper presented at the Eleventh International Conference Psychology of Mathematics Education. (Montreal).

LAWSON, A.E. y WOLLMAN, W.T., 1980. Developmental Level and Learning to Solve Problems of Proportionality in the Classroom, School Science and Mathematics, pp. 69-75.

LEINHARDT, G. 1987. The Development of an Expert Explanation: An Analysis of a Sequence of Subtraction Lessons, en L. Resrick (ed.), Knowing and Learning: Issues for a Cognitive Science of Instruction. (Lawrence ErIbaum Ass: Hillsdale, $\mathrm{NJ}$ ). 
LEINHARDT, G., 1988. Getting to Know: Tracing Students' Mathematical Knowledge from Intuition to Competence, Educational Psychologist, 23(2).

OSLOW, B., 1988. Overcoming Conceptual Obstacles: The Effectiveness of Conflict Discussion in the Mathematics Classroom. Paper presented at the Tenth Annual Meeting of North-American Chapter of International Group for the Psychology of Mathematics Education. (Dekalb).

PERRET-CLERMONT, A., 1984. La construcción de la inteligencia en la interacción social. (Visor: Madrid).

RESNICK, L., 1987. Constructing Knowledge in School, en L.S. Liben (ed.), Development and Learning: Conflict or Congruence? (Lawrence Erlbaum Ass.: Hillsdale, NJ).

RICCO, G., 1982. Les premières acquisitions de la notion de fonction lineair chez l'enfant de 7 à 11 ans, Educational Studies in Mathematics, 13, pp. 289-327.

ROMBERG, T., LAMMON, S. y ZARINNIA, A., 1988. The essencial features of the Mathematical Domain: Ratio and
Proportion (NCRMSE: Madison).

TOURNAIRE, F, 1986. Proportions in elementary school, Educational Studies in Mathematics, 17, pp. 40ذ-412.

TOURNAIRE, F. y PULOS, S., 1985. Proportional Reasoning: A Review of Literature. Educational Studies in Mathematics, 16, pp. 181-204.

VERGANAUD, G., 1982. Cognitive and developmental Psychology and Research in Mathematics Education: some Theorical and Methodological Issues, For the learning of Mathematics, 3(2), pp. 31-41.

VERGNAUD, G., 1983. Multiplicative Structures, en R. Lesh y M. Landau (eds.), Acquisition of Mathematics Concepts and Processes. (Academic Press: London).

WOOD, T. y YACKEL, E., 1990. The Development of Collaborative Dialogue within Small Group Interactions, en L.Steffe y T. Wood (eds.), Transforming Early Childhood Mathematics Education. (Lawrence Erlbaum Ass.: Hillsdale, NJ). 